\title{
EL DERECHO HUMANO AL AGUA POTABLE EN COLOMBIA: DECISIONES DEL ESTADO Y DE LOS PARTICULARES *
}

\author{
THE HUMAN RIGHT TO DRINKING WATER IN COLOMBIA: DECISIONS BY THE STATE AND \\ PRIVATE ACTORS
}

Judith Echeverria-Molina ${ }^{\text {a }}$

DOI: https://doi.org/10.11144/Javeriana.vj136.dhap

Universidad del Norte, Colombia

jechever@uninorte.edu.co

ORCID: http://orcid.org/0000-0001-7326-5577

Fecha de recepción: 10 Octubre 2016

Shirley Anaya-Morales

Universidad del Norte, Colombia

Fecha de aprobación: 10 Julio 2017

ORCID: http://orcid.org/0000-0001-5003-3044

Fecha de publicación: 30 Mayo 2018

\section{Resumen:}

Este artículo presenta reflexiones alrededor de las discusiones que se suscitan desde el pronunciamiento de la ONU A/64/L.63/ Rev.1, resolución del 26 de julio de 2010, que declara el agua potable y el saneamiento como un derecho humano esencial, lo cual genera para los Estados obligaciones exigibles por parte de los particulares. A partir de lo anterior, surge para los Estados miembro de las Naciones Unidas, entre ellos Colombia, la tarea de garantizar el servicio de acueducto con calidad y cobertura al $100 \%$ a todos sus habitantes pero, a la vez, el Gobierno enfrenta el reto de conservar las fuentes de agua, que son el insumo para garantizar los derechos que la ONU ha considerado exigibles para todo ser humano. Es por ello que se plantean en este artículo los interrogantes e inquietudes en torno a las tareas que deben emprender el Estado colombiano y los particulares para el cumplimiento de las directivas de la ONU, sin detrimento de las fuentes de agua y la sostenibilidad del medio ambiente.

Palabras clave: agua, agua potable, derecho humano.

\begin{abstract}
:
This paper provides some reflections on the discussions resulting from the UN declaration, A/64/L.63/Rev.1. It is a resolution dated July 26, 2010 whereby the UN enacts the drinking water and sanitation as an essential human right, which creates some obligations the private actors will demand the Member States to comply with. Based on the foregoing, the UN Member States, including Colombia, are now required to ensure a water supply with quality and $100 \%$ coverage to their inhabitants via an aqueduct. However, governments face, in turn, the challenge to preserve the water sources constituting the raw material for ensuring those rights the UN has enacted as claimable by all human beings. This way, this paper poses some questions and queries regarding the tasks to be undertaken by both the Colombian State and the private actors to comply with the UN directives, without detriment to the water sources and the environmental sustainability.
\end{abstract}

Keywords: water, drinking water, Human Right.

\section{SUMARIO}

I. EL PANORAMA DE COLOMBIA CON RESPECTO AL AGUA POTABLE. II. COLOMBIA Y EL PROTOCOLO FACULTATIVO DE LOS DERECHOS ECONÓMICOS, SOCIALES Y CULTURALES. III. POLÍTICA ESTATAL DE SUBSIDIOS. CONCLUSIÓN. REFERENCIAS BIBLIOGRÁFICAS.

Notas de autor 


\section{EL PANORAMA DE COLOMBIA CON RESPECTO AL AGUA POTABLE}

Actualmente la humanidad atraviesa la crisis mundial del agua, ${ }^{1}$ debido a que gran parte de los recursos hídricos del planeta se encuentran sometidos a altos niveles de contaminación en diversos Estados, a los efectos de la sobreexplotación de los recursos naturales, la superpoblación y la consecuente degradación medioambiental. ${ }^{2}$

Sin embargo, lo que parece ser propiamente una crisis de escasez de los recursos hídricos, bien sea en su estado natural o tratada para consumo humano, no es sino un problema de gobernabilidad y gobernanza del agua $^{3}$ que se suma a la problemática de distribución inequitativa del vital líquido.

Según el Segundo Informe de las Naciones Unidas sobre el desarrollo de los recursos hídricos en el mundo, "El agua, una responsabilidad compartida", la cantidad de agua dulce existente en el planeta, no obstante su porcentaje, es suficiente para cubrir las necesidades básicas de todos los seres humanos; este informe afirma que la insuficiencia de agua se debe, principalmente, a un abastecimiento ineficaz y no a un déficit del recurso. Este abastecimiento ineficaz es producto de la ineficiente gestión del recurso, de la corrupción, así como de la falta de instituciones enfocadas en el correcto tratamiento de la gestión del agua. Asimismo, contribuyen negativamente la indiferencia burocrática y la falta de inversión, tanto en capital humano competente como en infraestructuras físicas adecuadas. La escasez de agua y el aumento de la contaminación son desafíos de origen social y político, que se pueden afrontar modificando la demanda y el uso del agua, mediante la educación, una mayor sensibilización y mediante la reforma de las políticas hídricas. La crisis del agua radica, cada vez más, en el modo de regir el acceso y el control sobre los recursos hídricos y sus beneficios. ${ }^{4}$

El anterior panorama también se refleja en Colombia, porque el abastecimiento de agua potable, a pesar de constituir un servicio público domiciliario ${ }^{5}$ y un derecho humano fundamental, ${ }^{6}$ que debe proveerse de manera obligatoria a sus habitantes en las cantidades mínimas, ${ }^{7}$ que garanticen el respeto por la dignidad humana, no ha podido ser enteramente satisfecho en todo el territorio nacional, lo cual denota, más que la imposibilidad de abastecer del recurso a los sectores más vulnerables, una crisis en la gobernabilidad, gestión o gobernanza del agua ${ }^{8}$ como quiera que:

[1]a gestión del agua en Colombia ha girado más en torno a factores económicos y productivos, que han determinado ciertos esquemas institucionales, pero poco se han explorado alternativas desde el punto de vista social y cultural, a pesar de ser estos factores importantes a nivel de gobernabilidad.

Y es que:

[1]a crisis del agua es un fenómeno multifactorial que parte de asumir que las decisiones sobre cómo los recursos hídricos son protegidos, manejados, utilizados, asignados y conservados, son decisiones de gobierno, por lo cual se cree que la crisis del agua es en realidad una crisis de gobernabilidad. ${ }^{9}$

Del modo expuesto, el derecho fundamental al agua potable queda en manos de las políticas públicas y la fiscalidad del Estado colombiano, que acoge la postura según la cual el derecho al agua potable es un derecho social, garantizado solo hasta el máximo de los recursos que el Estado destine para tal fin, convirtiéndolo en promesa de derecho; apartándose de los lineamientos establecidos por la Corte Constitucional la cual:

[d] ominada por un cierto activismo judicial, ha venido desarrollando, con la conjunción de justicia constitucional y tutela, la doctrina del derecho innominado al mínimo vital, con el cual ha contribuido decisivamente a iniciar la construcción de un Estado social de derecho en Colombia, es decir, hacia la realización plena de los derechos económicos, sociales y culturales. 
No obstante que el derecho al agua potable constituya un derecho social ${ }^{10}$, lo cierto es que en Colombia es un derecho fundamental, y como cualquier derecho, es exigible, ya en términos mediatos o inmediatos, y su justiciabilidad es una dimensión, la más importante sin duda, de su exigibilidad. ${ }^{11}$

Valido es mencionar entonces que en Colombia "existen esfuerzos relacionados con el agua como elemento constitutivo del Estado, riqueza de la nación, servicio público y derecho colectivo y del ambiente, con base en la Constitución Política de 1991 esta no define el agua como derecho humano fundamental, a pesar del reconocimiento hecho por Naciones Unidas en el Comentario General Número 15 de 2002" 12, como tampoco existe dentro del ordenamiento normativo colombiano disposiciones que establezcan el derecho humano al agua, salvo las innumerables veces en que la Corte Constitucional se ha pronunciado sobre el mínimo vital de agua potable, lo cual ha generado una importante doctrina al respecto ${ }^{13}$.

Así entonces se pueden contrastar dos posturas en torno al acceso al agua potable en nuestro país: una de desarrollo legal y jurisprudencial que garantiza el agua potable a sus nacionales y la otra postura representada en las acciones del estado rodeadas de problemáticas de gobernanza y gobernabilidad sobre la base del agua potable como derecho social.

Ahora, en relación con los actores que intervienen en el abastecimiento del vital líquido:

[e]n la legislación colombiana se ha considerado el agua como un bien nacional de uso público, por ser este un recurso natural indispensable para la vida y el desarrollo económico del país. Aunque las principales normas y políticas se han basado en este principio desde la Constitución de 1886, ello no ha impedido que el sector privado juegue un papel importante en la provisión de los servicios de agua potable, saneamiento básico y generación de energía. ${ }^{14}$

Lo anterior permite vislumbrar que la evidente participación de empresas privadas en la gestión y operación de los servicios públicos domiciliarios en el país, incluyendo el servicio de acueducto, no exime al Estado de la tarea de garantizar los servicios públicos de agua potable y saneamiento básico.

Por tanto, se presentan estas reflexiones en torno a las tareas que el Estado colombiano en un futuro próximo deberá realizar y que deben buscar la garantía del derecho al agua potable para todos sin que se merme el recurso natural, sin que se afecte la calidad de las fuentes, sin que se genere desperdicio y sin que se afecten las finanzas del Estado.

Desde una perspectiva general, es necesario reflexionar sobre lo que afirma González ${ }^{15}$ cuando asevera:

[1]a vida en el planeta pende de un hilo. Nos estamos quedando sin reservas de agua potable. Más del $70 \%$ de la tierra está cubierto de agua. A pesar de su abundancia en los mares y océanos, el 97,5\% de toda el agua del orbe es salada, solo el 2,5 es dulce.

Esto lleva a considerar que si bien existe un derecho a un mínimo vital, también existe una

Esto lleva a considerar que si bien existe un derecho a un mínimo vital, también existe una limitación en cuanto a la existencia y disponibilidad del recurso, que está distribuido de manera inequitativa ${ }^{16}$ y que se ve afectado por el aumento de la población y a la vez por el consumo de los individuos. ${ }^{17}$

Esta población creciente también ha cambiado sus hábitos de consumo y cada día, por necesidades creadas o por hábitos de higiene, la humanidad cuenta con un consumo promedio mayor que en épocas anteriores. Por tal razón cabe preguntar qué se entiende por mínimo vital de agua, ${ }^{18}$ ya que si bien existe un derecho, es necesario determinar si lo mínimo es coincidente con lo básico y si ese mínimo es a todos los habitantes del país o si solo son para aquellos que se encuentren en condiciones vulnerables. Informes indican que:

Hacia el año 2025 se estima que mil ochocientos millones de personas vivirán en regiones con escasez de agua y dos tercios de la población mundial residirán bajo condiciones de estrés hídrico. Esto dirigirá los conflictos hacia la competencia por el agua y propiciará enfermedades debidas a alteraciones en la calidad del agua, así como también se experimentarán pérdidas de biodiversidad y de los servicios de los ecosistemas. ${ }^{19}$ 
En este caso, no hay que olvidar la doble connotación que tiene el agua: por un lado es un recurso natural y por el otro un derecho. Como recurso natural se encuentra en peligro en virtud de la crisis ambiental y del estrés hídrico ${ }^{20}$ que se vive en algunos países.

Ante este panorama de escasez, estrés hídrico y excesivo consumo, vale la pena preguntarse si, ¿̇asumir el costo de la cobertura del agua potable a la población excluida, será una acertada decisión que conlleve a la justiciabilidad del derecho de acceso al agua potable por parte del Gobierno colombiano? ¿Será esta acción la necesaria para la sociedad colombiana que sufre los estragos de carecer total o parcialmente del vital líquido, o que pudiendo acceder a él, no se encuentra en condiciones para consumo humano?

Algo importante en relación con las responsabilidades es que "la no exigibilidad del logro inmediato no libera a los Estados de la existencia de las obligaciones", ${ }^{21}$ lo que implica que es un deber del día a día incluir en los presupuestos nacionales, departamentales y municipales los rubros que garanticen la ampliación de la cobertura, junto con la calidad, frecuencia y continuidad del servicio, pero que hasta la fecha tampoco han garantizado el $100 \%$ de cobertura, a pesar de los esfuerzos de la nación y de las entidades territoriales.

\section{COLOMBIA Y EL PROTOCOLO FACULTATIVO DE LOS DERECHOS ECONÓMICOS, SOCIALES Y CULTURALES}

En la década de los noventa el Comité de Derechos Económicos, Sociales y Culturales (DESC) ${ }^{22}$ planteaba la redacción de un Protocolo Facultativo del Pacto Internacional de Derechos Económicos, Sociales y Culturales (PIDESC) que, en esencia, permitiera la interposición de quejas individuales de los particulares por la falla del Estado a propósito de los derechos DESC. En este contexto, en el seno del Comité DESC se empezó a elaborar un Proyecto de Protocolo Facultativo que vio la luz en diciembre de 1996 y se sometió a consideración de la Comisión Interamericana de Derechos Humanos:

A partir de ese momento se articuló una estructura de trabajo orientada a la definitiva redacción del protocolo, que se
desenvolvió a través de lo que pueden considerarse distintas fases. En primer lugar, a través de la Comisión de Derechos
Humanos; después mediante el Consejo de Derechos Humanos -que sustituyó a la Comisión, y con el nombramiento de un
experto independiente que evaluara la cuestión. Finalmente, cuando parecía haberse alcanzado el necesario consenso político
al respecto, en 2002 se creó un Grupo de Trabajo de composición abierta presidido por Catarina de Albuquerque, ${ }^{23}$ que
se encargó de configurar y aprobar definitivamente el Proyecto de Protocolo Facultativo en 2008. Tras ello, el Consejo de
Derechos Humanos adoptó dicho Proyecto de Protocolo Facultativo, instando su aprobación por la Asamblea General, en
la 66. a sesión, que tuvo lugar el 10 de diciembre de $2008 .{ }^{24,25}$

De acuerdo con la Oficina Regional del Alto Comisionado de las Naciones Unidas para los Derechos Humanos en América del Sur, el Protocolo Facultativo de los Derechos Económicos, Sociales y Culturales 26 es:

Un mecanismo que permite que personas o grupos presenten quejas ante el Comité de Derechos Económicos, Sociales y Culturales de Naciones Unidas, respecto de violaciones de estos derechos cometidas por un Estado Parte. Por ejemplo, interferir indebidamente en el goce de un derecho, no adoptar medidas dirigidas a su realización, denegar derechos de manera discriminatoria o adoptar medidas deliberadamente regresivas sin justificación adecuada. Es más, se podrá presentar una comunicación directamente ante el Comité DESC. El Protocolo Facultativo establece tres procedimientos internacionales de protección: comunicaciones individuales (quejas o peticiones), comunicaciones interestatales y procedimiento de investigación de violaciones graves o sistemáticas de los DESC. ${ }^{27}$

Colombia aún no ha decidido dar ese gran paso, que le permitiría cumplir con la obligación de proteger los derechos económicos sociales y culturales, fundamentalmente el derecho al agua potable. La importancia de suscribir y ratificar el protocolo facultativo, recae en que este garantizará en mayor medida la justiciabilidad de los derechos humanos para toda una nación. 
Colombia firmó el Pacto Internacional de Derechos Económicos Sociales y Culturales el 23 de marzo de 1976; al día de hoy es fundamental para toda la población colombiana que el Estado firme y ratifique el Protocolo para que constituya una herramienta que quizá permita una mayor exigibilidad en relación con derechos humanos como el de acceso al agua potable.

Sin embargo, el Protocolo, ya ante las particularidades del recurso (agua) y del servicio (acueducto), nos plantea el siguiente interrogante: ¿Qué tan beneficioso resulta para el medio ambiente y del agua en su estado natural la firma de este instrumento y si este instrumento entraría a llenar un vacío en la gran lucha por defender los derechos económicos, sociales y culturales? Esto nos conduce a otro cuestionamiento: gracias a la denuncia internacional individual y colectiva del PIDESC, ¿permitiría resolver casos más concretos y exigir a sus Estados que cumplan con las sentencias que imparta el Comité DESC? Pero queda la duda sobre la sostenibilidad de las infraestructuras y del medio ambiente.

No hay que perder la perspectiva de los límites a este derecho. Es importante recalcar que no es agua para todo consumo de una persona, ${ }^{28}$ al igual que no debe ser para todas las personas, ya que esta garantía de gratuidad o de subsidio, debe ser para aquellos en situaciones de vulnerabilidad.

Asimismo, el derecho humano al agua debe ser considerado conjuntamente con otros derechos consagrados en la Carta Internacional de Derechos Humanos, como la intimidad o la vivienda, entre los que ocupa un lugar primordial el derecho a la vida y a la dignidad humana, vinculación que no hace sino ampliar el espectro del derecho de aguas. Nos hallamos ante un concepto que supera claramente la limitación a la satisfacción de las necesidades esenciales de subsistencia, comprendiendo, además, el agua necesaria para satisfacer otras necesidades como requisito previo para asegurar el ejercicio de otros derechos humanos. 29

Vale recordar que en Colombia en 2007, se presentó por iniciativa ciudadana, un referendo del agua que fue archivado porque:

[e]l Gobierno de turno se opuso, tras considerar dicho referendo como regresivo e idealista. Según el Gobierno, el sistema de subsidios cruzados del esquema tarifario que opera en Colombia es equiparable con un mínimo vital de $14 \mathrm{~m}^{3} /$ hogar para el estrato 1 y $8 \mathrm{~m}^{3} /$ hogar para el estrato 2 ; por tanto, la propuesta de mínimo vital $\left(6-8 \mathrm{~m}^{3} / \mathrm{hogar}\right)$ del referendo del agua no favorecía a los hogares con mayores carencias, pero sí beneficiaría a los estratos más altos, porque antes de la nueva ley los estratos 5 y 6 no tenían subsidios y después de la ley recibirían el mínimo vital. ${ }^{30,31}$

Es más, con la aprobación de un mínimo vital, los usuarios ubicados en viviendas de categoría o estratos 5 y 6 se beneficiarían, ya que no tendrían sobreprecio por el consumo básico que sería gratuito. En este caso, el Estado daría subsidios o descuentos a quienes están en capacidad de pagar por el servicio de acueducto.

Lo anterior entraría a perjudicar al Estado, porque le correspondería, en este caso, aportar más recursos para subsidios, no solo para usuarios de menores ingresos sino a todos los habitantes en general, con la consecuencia de perder ese sobreprecio que hoy se cobra a los usuarios que los estratos altos aportan en su factura.

Bajo estos argumentos el Estado tenía razón ya que si miramos la estructura de tarifas prevista en la Ley 142 de 1994, existe un sistema de subsidios cruzados que afecta con un sobreprecio, como ya se mencionó, a los usuarios residenciales de mayores ingresos y a los usuarios comerciales e industriales, para que este rubro sea distribuido entre los usuarios de menores ingresos del sector residencial.

Ante este escenario solo quedaría para los usuarios de menores ingresos el sobreprecio de los sectores industrial y comercial, que por obvias razones no entraría a ser subsidiado por el Estado.

Se resalta que el sobreprecio a los usuarios de más altos ingresos se hace sobre el consumo y si se establece un mínimo gratuito a todos, se pierde para los usuarios de menores ingresos y para el Estado ese sobreprecio, ya que no todos los usuarios de mayores ingresos manejan altos consumos.

Es decir,

[1]os pobres recibirían el mínimo vital a cambio de perder los subsidios. Ya que los recursos públicos son limitados, se requerirían sacrificios en la expansión de la cobertura, para destinar recursos a la financiación de un mínimo vital, que beneficiaría principalmente a los más ricos. Pero la realidad es que con el sistema de subsidios, un hogar del estrato 1 no recibe 
$14 \mathrm{~m}^{3}$ de agua potable sin cobro (ni $8 \mathrm{~m}^{3}$ de agua potable al estrato 2). Como el subsidio es simplemente un porcentaje de descuento en la tarifa de los primeros $20 \mathrm{~m}^{3}$ de agua potable consumida, los hogares deben pagar una factura, así su consumo sea inferior a este supuesto mínimo vital. ${ }^{32}$

Otra de las decisiones que se contempla en esta reflexión, a partir de las consideraciones de la ONU es aquella en la que el Estado Colombiano asuma el costo de la cobertura de agua potable de la población excluida de este derecho fundamental -servicio público domiciliario-, pero realmente esta es una obligación estatal incluida en todos los planes y proyectos del Estado, al menos del colombiano.

En este sentido se observa que las personas realmente pobres, los que viven con menos de dos dólares diarios, les es imposible pagar el costo que genera la prestación de este servicio y de cualquier otro.

¿La razón? No tienen con qué pagar. En esta situación se encuentra más de la mitad de la población mundial según datos las Naciones Unidas. Por ejemplo en Barranquilla, Colombia, el precio promedio del agua del servicio público es de US\$ 0,55 por metro cúbico. En cambio, el mismo metro cúbico de agua cuesta a los que se surten de carro tanques y transportistas de agua US\$ 5,50. ${ }^{33}$

En Colombia hay una clara política de subsidios y de fijación de los consumos subsidiables ${ }^{34}$ que se acerca al $50 \%$ sobre el consumo básico, ${ }^{35}$ consumo que garantiza las necesidades esenciales de cualquier persona en el país.

En Sentencia T-055 de 2011, la Corte Constitucional manifestó:

Dentro del marco jurídico constitucional de la figura de Estado social de derecho adoptada por la Constitución de 1991 ,
el texto Superior dispuso en sus artículos 365 a 370 que los servicios públicos son factores esenciales y de gran importancia
para materializar la función del Estado. Así, la eficiencia, la oportunidad y el mayor cubrimiento de los servicios públicos,
son los medios más adecuados que tiene el Estado para asegurar el mejoramiento de la calidad de vida de todas las personas y
dar solución a las necesidades básicas insatisfechas en materia de saneamiento ambiental y de agua potable, de tal suerte que
muchos de los factores que regulan legalmente su prestación deben tener como consideración fundamental el interés social
que ellos revisten. Es a partir de este criterio general de cubrimiento a toda la población que la misma Carta delegó en la ley,
la función reguladora de tales servicios públicos, estableciendo que los mismos pueden estar a cargo del Estado de manera
exclusiva o indirectamente por comunidades organizadas o por particulares. ${ }^{36}$

Es importante destacar, que en la Sentencia anteriormente citada se manifiesta que la razón de ser de los servicios públicos y la necesidad de su regulación por parte del Legislador se regirán siempre por la necesidad de asegurar su prestación eficiente a todos los habitantes del territorio nacional, es decir, sin importar su nivel de ingresos.

Adicionalmente, el estado reservó la tarea de vigilar y controlar a las empresas y prestadores de los servicios públicos domiciliarios, tal como lo prevé el artículo 370 de la Carta Política, al crearse la Superintendencia de Servicios Públicos Domiciliarios.

Las líneas anteriores se enfocan en el deber ser del Estado Colombiano en relación con los servicios públicos domiciliarios. Ahora, si nos centramos específicamente en el servicio de agua potable, su importancia es doble, como quiera que es también un derecho fundamental; en efecto, desde sus inicios la Corte Constitucional ha considerado que en virtud de lo dispuesto por la Constitución en el artículo 366, permitir el acceso al agua potable y al saneamiento básico, además de ser un objetivo fundamental como Estado social de derecho y un medio para asegurar el desarrollo y realización de necesidades básicas insatisfechas, ha adquirido la connotación de derecho fundamental para todas las personas.

La Corte afirma que son numerosos los argumentos jurídicos que permiten considerar el derecho al agua potable como un derecho fundamental (cuando está destinada al consumo humano; cuando ella es necesaria para preservar la vida, la salud o la salubridad de las personas; cuando existe afectación particular del derecho fundamental en cabeza de una, varias o múltiples personas o cuando existe la amenaza de consumación de un perjuicio irremediable en la órbita de este derecho fundamental ${ }^{37}$ ) y de esto no hay duda, muy particularmente y cuando por medio de este y su acceso efectivo con la prestación del servicio de acueducto se 
está dando alcance a uno de los fines esenciales del Estado: la solución de las necesidades básicas insatisfechas de las personas y porque con la garantía de este derecho se protegen otros derechos fundamentales tan trascendentales como la vida en condiciones dignas y la salud.

\section{POLÍTICA ESTATAL DE SUBSIDIOS}

Las anteriores son motivaciones suficientes para que el Estado colombiano independientemente de las directivas de la ONU, siga incluyendo en los presupuestos de la nación, de los departamentos y de los municipios las asignaciones para ampliar la cobertura de los servicios públicos de agua potable y saneamiento, recursos que se convierten en subsidios a la oferta de dichos servicios y que se verán reflejados en un descuento en la tarifa a los usuarios de menores ingresos; (es decir, de los estratos 1, 2 y 3), lo que significa, que para este sector "tienen como fin garantizar que el prestador mantenga el equilibrio financiero, entre los usuarios que pagan una tarifa y las empresas que mediante el recaudo prestan el servicio, lo que debe garantizar la recuperación de sus costos económicos" ${ }^{38}$

Sin embargo, no es tarea sencilla, se puede afirmar que es una labor altamente compleja, ya que se requiere del mayor esfuerzo del Gobierno colombiano, pero sobre todo, se trata de un cambio de perspectiva, de una trasformación en la visión de este recurso. Se requiere cambiar el paradigma, del agua potable de invertir partidas en el subsidio de los de mayor capacidad adquisitiva sin que la sociedad reciba a cambio el control sobre el consumo ni la contaminación indiscriminada y de esta manera empezar a salir de la deficiente gobernabilidad del agua que impide la justiciabilidad de este esencial derecho.

En este punto vale la pena traer la pregunta de Barlow: "¿A quién se le debe dar el poder de abrir y cerrar el grifo: a la gente, a los gobiernos o a la mano invisible del mercado?". 39

Lo anterior porque para el caso de los Estados, les exigimos que hagan las inversiones que les son reclamadas, pero, ¿quién controla el consumo y sobre todo el exceso el consumo de agua y su contaminación?

El concepto de mínimo vital descansa entonces sobre la idea de garantizar las condiciones mínimas de subsistencia concretas para que las personas puedan ejercer sus capacidades y desenvolverse en la sociedad. Y por capacidades podemos entender las relaciones humanas, el espacio, los talentos, sentimentalidad y el contexto en el que se exigen o defienden los derechos. Pero no solo desde el punto de vista de exigencia de los derechos humanos o de los derechos constitucionales, sino también desde los deberes humanos, de las relaciones existentes en un ámbito de no satisfacción de los derechos y los cuales pueden ser satisfechos total o parcialmente, por varios círculos, dependiendo del grado de cercanía y relación con el caso. ${ }^{40}$

En este orden de ideas, la Corte Constitucional de Colombia, Sentencia T-581A ha establecido que el derecho fundamental al mínimo vital debe ser evaluado enfocándolo desde la satisfacción de las necesidades mínimas del individuo, por lo cual es necesario realizar una evaluación de las circunstancias de cada caso concreto, haciendo una valoración que se encamine más hacia lo cualitativo que a lo cuantitativo, verificándose que quien alega su vulneración tenga las posibilidades de disfrutar de la satisfacción sobre los derechos de vivienda y recreación, como mecanismos para hacer realidad su derecho a la dignidad humana de necesidades como la alimentación, el vestuario, la salud y la educación. ${ }^{41}$ En este caso, se indica que el operador o empresa tiene el deber de evaluar los casos en los que las personas por distintas razones no pueden pagar el servicio de agua potable.

Volviendo a la pregunta de Barlow sobre el papel del Estado en el control del consumo vale la pena reflexionar ya que "hoy en día, las pérdidas de agua llegan a representar entre el 30 y el $50 \%$ del total del consumo. ${ }^{42}$ En este caso, si hubiera controles a los usuarios y mayores sanciones a los operadores y empresarios del agua, esas pérdidas del líquido podrían favorecer a otros usuarios y al Estado aplazar inversiones, que siempre afectarán las tarifas u otras inversiones estatales, ya que con esos recursos se podría subsidiar ${ }^{43} \mathrm{o}$ financiar más oferta y demanda, ${ }^{44}$ lo cual se evidencia en un menor costo de la tarifa. 
Así las cosas, al Estado colombiano se le exigen las decisiones y acciones contempladas en las anteriores reflexiones en torno al agua potable, pero además a los particulares, usuarios o titulares de este derecho, sobre quienes también recae la responsabilidad de usar el preciado líquido en las proporciones, medidas y el cuidado que demanda un recurso limitado como el agua.

\section{CONCLUSIÓN}

El presente trabajo estuvo dirigido a reflexionar sobre las decisiones y acciones que surgen para el Estado y los particulares dirigidas hacia una mejor gestión del agua potable, a partir de la declaratoria de este recurso como derecho humano y las posibilidades reales que tiene el Estado colombiano para cumplir, en el menor tiempo posible con las mejores condiciones de suministro de agua potable para sus habitantes.

En primer lugar, nos centramos en analizar la gestión del agua y sus falencias; cuya solución, según diversas posturas, recae en adoptar una nueva perspectiva de gobernabilidad y gobernanza de este recurso, para que se logre un mayor compromiso de los gestores y beneficiarios del recurso, para romper el círculo que actualmente existe de esperar que el Estado es el único que tiene el deber de conservación y cuidado, para lograr una política responsable con el medio ambiente y que tienda al control del consumo y no solo a la garantía del recurso.

Se contempló la posibilidad de que el Estado ratifique el Protocolo facultativo de derechos económicos, sociales y culturales como una decisión que conllevaría a que el agua, como derecho, tenga una garantía de justiciabilidad en el ámbito internacional, sin perder de vista los límites de este derecho; que como se ha expresado en el texto genera enormes compromisos para la comunidad, el Estado y los particulares, sobre todo a aquellos que reciben los servicios públicos (en este caso el acueducto) con un fuerte componente de subsidios.

Así, a partir de tales reflexiones, se abre la invitación a que el Estado, los particulares, los usuarios y los titulares del derecho al agua, se concienticen sobre los retos que demanda la gestión del líquido para ser eficaz y eficiente, y el papel que cada uno ejerce como engranaje social que debe orientar todo el esfuerzo, tanto individual como colectivo, a que la administración del recurso agua sea optimizado, respetado y garantizado de acuerdo con las prerrogativas tratadas en el presente trabajo.

\section{Agradecimientos}

Este artículo surge del proyecto de Investigación denominado "El derecho Humano al agua”, el cual fue desarrollado al interior de la Maestría en Derecho bajo la tutoría de la Profesora Judith Echeverría Molina.

\section{Referencias Bibliográficas. Libros}

AMADOR CABRA, LUIS. Los servicios públicos frente a las reformas económicas en Colombia. 410 (Universidad Externado de Colombia, Bogotá. 2011).

BARLOW, MAUDE, El derecho al agua. Agua, un derecho y no una mercancía. Propuestas de la sociedad civil para el modelo público de agua 109 (Icaria, Barcelona, España, 2009).

GUERRERO, MANUEL \& SHIFTER, ISAAC, La huella del agua 63 (Fondo de Cultura Económica, México, 2012). OSORIO SIERRA, Álvaro, EMBID IRUJO, ANTONIO \& HURTADO MORA, JORGE IVÁN, Régimen de los usos del agua. Análisis desde la perspectiva Jurídica, 87-88 (Universidad de Zaragoza-Universidad Externado de Colombia, Zaragoza, Bogotá, 2016).

RODRÍGUEZ FERNÁNDEZ, SAMUEL, La tutela penal del agua. El bien jurídico protegido, 88 (Dykinson, Madrid, España, 2009). 
SALAMERO TEIXIDÓ, LAURA, La protección de los derechos sociales en el ámbito de las Naciones Unidas, 55 (Aranzadi, Civitas-Thomson Reuters, Pamplona, España, 2012).

\section{Referencias Bibliográficas. Revistas}

AYEB, HABIB, Agua y poder. Geopolitica de los recursos hidráulicos en Oriente Próximo, Bella Terra 13 (2001).

BOHÓRQUEZ CALDERA, LUIS, Bioética del derecho al agua potable. Revista Ciencias Sociales, 15, 2, 289. (2015).

GÓMEZ BUSTOS, IVETTE, La acción colectiva del agua en Colombia y el referendo como acercamiento de democracia directa, Revista análisis político, 80 (2014).

GONZÁLEZ GONZÁLEZ, JORGE, El acceso al agua potable como derecho humano. Su dimensión internacional, Club Universitario, 27, 133 (2014).

HERNÁNDEZ, HUGO ALFONSO \& MÉNDEZ SAYAGO, JHON, Viabilidad económica del mínimo vital de agua potable en la ciudad de Bogotá D. C. Revista Producción + Limpia, 8, 1, 104 (Enero-Junio 2013).

JIMÉNEZ RAMÍREZ, MILTON CÉSAR, El minimo vital de acceso al agua potable frente a las empresas de servicios públicos en Colombia: personas en situación de debilidad manifiesta vs mercado de servicios públicos, Revista Pensamiento Jurídico, 38, 117 (2013).

MIGUEZ CARAMÉS, DIANA MARGARITA, Gestión integrada de recursos hidricos en Uruguay en el contexto internacional, Revista del Laboratorio Tecnológico del Uruguay, 72 (2015).

MOTTAVARGAS, RICARDO, El derecho al agua potable en la jurisprudencia colombiana, Revista Republicana, 66 (2011). Disponible en: http://www.corteidh.or.cr/tablas/R21767.pdf

PÉREZ DE LOS COBOS, ELISA, La defensa del derecho al agua como derecho Colectivo desde su perspectiva ambiental, Revista Catalana de Derecho Ambiental VI, 2, 11. (2015).

ZAMUDIO RODRÍGUEZ, CARMEN, Gobernabilidad sobre el recurso hidrico en Colombia: entre avances y retos, Revista Gestion Ambiental, 15 (2012). Disponible en: https://revistas.unal.edu.co/index.php/gestion/article/v iew/36284/42930

ZÚNIIGA, FRANCISCO, Derechos económicos, sociales y culturales: apuntes acerca de la naturaleza y justiciabilidad de los derechos fundamentales. Revista Pensamiento Constitucional, Pontificia Universidad Católica del Perú, 335 (2008).

\section{Referencias Bibliográficas. Documentos}

ABRAMOVICH, VÍCTOR \& COURTIS, CHRISTIAN, Hacia la exigibilidad de los derechos económicos, sociales y culturales. Estándares internacionales y criterios de aplicación ante los tribunales locales, 11 (1997). Disponible en: http://miguelcarbonell.com/artman/uploads/1/Exigibilidad_de_los_DESC_-_Abramovich.pdf.

CARDONA LÓPEZ, ALBERTO, Consideraciones sobre el sector de agua potable y saneamiento básico en Colombia, 4 (2010). Disponible en: https://colaboracion.dnp.gov.co/CDT/Estudios\%20Econmicos/Consideraciones\%20 sobre\%20el\%20Sector\%20de\%20agua\%20potable\%20y\%20saneamiento\%20b\%C3\%A1sico.pdf, 4 (2010).

RED-DESC. Sección 1: La historia del PF-PIDESC, 4 (2010). Disponible en: https://www.escr-net.org/es/recurso s/seccion-1-historia-del-pf-pidesc

TELLO, LUISA FERNANDA, El acceso al agua potable, ¿un derecho humano? Disponible en: http://www.corteidh .or.cr/tablas/R21767.pdf

UN, Agua para todos, agua para la vida (2003). Disponible en: http://www.un.org/esa/sustdev/sdissues/water/WW DR-spanish-129556s.pdf

$\mathrm{UN}$, El agua, una responsabilidad compartida. $2^{\circ}$ Informe de las Naciones Unidas sobre el desarrollo de los recursos hidricos en el mundo, 1-52 (2006). Disponible en: http://unesdoc.unesco.org/images/0014/001444/144409S.pdf

UN, Derechos humanos. Disponible en: http://www.un.org/es/rights/overview/ 
UN, Protocolo Facultativo del Pacto Internacional de Derechos Económicos, Sociales y Culturales, A/RES/63/117 Resolución de la Asamblea General de 10 de diciembre de 2008, Disponible en: http://www.un.org/en/ga/searc h/view_doc.asp?symbol=A/RES/63/117\&Lang=S

UN, Sra. Catarina de Albuquerque, Relatora Especial sobre el derecho humano al agua potable y al saneamiento. Disponible en: http://www.ohchr.org/SP/Issues/WaterAndSanitation/SRWater/Pages/CatarinaDeAlbuque rque.aspx (n. d.).

\section{Referencias Bibliográficas. Jurisprudencia colombiana}

Colombia, Corte Constitucional, Sala Plena, Sentencia T-581A de 2011, Expediente T-3.011.626, magistrado ponente: Mauricio González Cuervo.

Colombia, Corte Constitucional, Sala Plena, Sentencia T-055 de 2011, Expediente T-2.804.492, magistrado ponente: Jorge Iván Palacio Palacio.

Colombia, Corte Constitucional, Sala Plena, Sentencia T-546 de 2009, Expediente T-2259519, magistrada ponente: María Victoria Calle Correa.

Colombia, Corte Constitucional, Sala Plena, Sentencia T-888 de 2008, Expediente T-1.822.669, magistrado ponente: Marco Gerardo Monroy Cabra.

\section{Referencias Bibliográficas. Normatividad colombiana}

Colombia, Ley 142 de 1994, por la cual se establece el régimen de los servicios públicos domiciliarios y se dictan otras disposiciones, 41.433 Diario Oficial, 11 de julio de 1994. Disponible en: http://www.alcaldiabogota.gov.co/si sjur/normas/Norma1.jsp?i=2752

CRA, Clasificación temática. Consumo básico de agua (2016). Disponible en: http://www.cra.gov.co/es/novedades /noticias/23739-conoce-el-nuevo-consumo-b

\section{Notas}

* Artículo de investigación.

1 El Informe de las Naciones Unidas sobre el Desarrollo de los Recursos Hídricos en el Mundo "Agua para todos, agua para la vida", ha indicado que: "La Tierra, con sus diversas y abundantes formas de vida, que incluyen a más de seis mil millones de seres humanos, se enfrenta en este comienzo del siglo veintiuno con una grave crisis del agua. Todas las señales parecen indicar que la crisis se está empeorando y que continuará haciéndolo, a no ser que se emprenda una acción correctiva. Se trata de una crisis de gestión de los recursos hídricos, esencialmente causada por la utilización de métodos inadecuados. La verdadera tragedia de esta crisis, sin embargo, es su efecto sobre la vida cotidiana de las poblaciones pobres, que sufren el peso de las enfermedades relacionadas con el agua, viviendo en entornos degradados y a menudo peligrosos, luchando por conseguir una educación para sus hijos, por ganarse la vida y por solventar a sus necesidades básicas de alimentación". UN, Agua para todos, agua para la vida (2003). Disponible en: http://www.un.org/esa/sustdev/sdissues/water/WWDR-spanish-129556s.pdf

2 LUISA FERNANDA TELLO, El acceso al agua potable, ¿un derecho humano? Disponible en: http://www.corteidh.or.cr/ tablas/R21767.pdf.

3 Sobre este aspecto es importante resaltar que los conceptos gobernanza y gobernabilidad del agua son tratados como sinónimos y en realidad son distintos y apuntan a aspectos relacionados, el primero con la estructura política administrativa para la gestión política del agua y el segundo con la integración de los actores, gestores, y empresarios, para el logro de sinergias para la administración y conservación del servicio.

$4 \mathrm{UN}$, El agua, una responsabilidad compartida. $2^{\circ}$ Informe de las Naciones Unidas sobre el desarrollo de los recursos hídricos en el mundo, 1-52 (2006). Disponible en: http://unesdoc.unesco.org/images/0014/001444/144409S.pdf 
5 La Ley 142 de 1994 establece en su artículo 1 que "Esta Ley se aplica a los servicios públicos domiciliarios de acueducto, alcantarillado, aseo, energía eléctrica, distribución de gas combustible, telefonía [fija] pública básica conmutada y la telefonía local móvil en el sector rural; a las actividades que realicen las personas prestadoras de servicios públicos de que trata el artículo 15 de la presente Ley, y a las actividades complementarias definidas en el Capítulo II del presente título y a los otros servicios previstos en normas especiales de esta Ley".

6 Corte Constitucional de Colombia, Sala Plena, Sentencia T-888 de 2008, Expediente T-1.822.669, magistrado ponente: Marco Gerardo Monroy Cabra.

7 El mínimo vital se define como un derecho fundamental innominado o derecho a la subsistencia, y esto surge de la interpretación holística de la Constitución Política y del Bloque de Constitucionalidad. Los mínimos vitales son un mínimo de condiciones materiales necesarias para llevar a una existencia digna, garantizadas a su vez por la observancia de otros derechos. En esta situación el derecho al mínimo vital de agua hace parte del principio de progresividad, regulado en el Protocolo Internacional de San Salvador. La progresividad exige a las autoridades estatales desarrollar gradualmente los derechos económicos, sociales y culturales para corregir graves desigualdades sociales y promover mejores condiciones de vida, entre ellas brindar agua potable a la totalidad de la población como un bien de uso público. RICARDO MOTTA, El derecho al agua potable en la jurisprudencia colombiana, Revista Republicana, 66 (2011). Disponible en: http://www.corteidh.or.cr/tablas/R21767.pdf

8 Frente a la gobernabilidad del agua, el Programa de las Naciones Unidad para el Desarrollo, la define como el conjunto de sistemas políticos, sociales, económicos y administrativos encargados de desarrollar y gestionar los recursos hídricos y su distribución. La gobernabilidad del agua cubre así mismo un conjunto de temas íntimamente ligados al agua, desde la salud y la seguridad alimentaria hasta el desarrollo económico, el uso de la tierra y la preservación del entorno natural del que dependen nuestros recursos de agua. La Gobernabilidad del Agua se preocupa por diseñar y adoptar las leyes, las políticas y las instituciones adecuadas. Finalmente, clarifica los roles y las responsabilidades de todos los actores- gobiernos locales y nacionales, el sector privado y la sociedad civil- en lo que a la propiedad y a la administración de los recursos en agua concierne". CARMEN ZAMUDIO RODRÍGUEZ, Gobernabilidad sobre el recurso hidrico en Colombia: entre avances y retos, Revista Gest. Ambient., 15, 3, 99-112 (2012). Disponible en: https://revistas.unal.edu.co/index.php/gestion/article/view/36284/42930 Además, la gobernabilidad del agua se refiere a la gama de sistemas políticos, sociales, ambientales, económicos y administrativos que existen para regular el desarrollo y la gestión de los recursos hídricos y el suministro de servicios asociados al agua. También se reconoce como el conjunto de sistemas para participar en la toma de decisiones sobre la gestión del agua y la prestación de servicios. En última instancia, la gobernabilidad del agua determina quién obtiene qué, cómo y cuándo. Igualmente, los sistemas de gobernabilidad del agua reflejan realidades políticas y culturales de orden nacional, regional y local. La gestión efectiva del agua busca un equilibrio entre las dimensiones social, económica, política y ambiental (WGF, 2010). De tal manera, que los componentes de la gobernabilidad del agua giran en torno a tres pilares estructurales: - los marcos normativos y legislativos para proteger los recursos hídricos y garantizar agua para el desarrollo social y económico; - las instituciones para la gestión del agua que faciliten la participación de todos los interesados de forma transparente y de manera responsable; y - los mecanismos y reglamentos para la toma de decisiones que permitan un uso responsable del poder político, óptimo uso de los recursos, el desarrollo sostenible y la sostenibilidad ecológica. $\mathrm{Al}$ mismo tiempo, estos tres elementos fundamentales se encuentran integrando de manera horizontal, cuatro sectores: agua para la gente, agua para comida, agua para la naturaleza y agua para la industria y otros usos. CARMEN ZAMUDIO RODRÍGUEZ, Gobernabilidad sobre el recurso hidrico en Colombia: entre avances y retos, Revista Gest. Ambient., 15, 3, 101-102, 106 (2012).

9 FRANCISCO ZÚNIIGA, Derechos económicos, sociales y culturales: apuntes acerca de la naturaleza y justiciabilidad de los derechos fundamentales, Revista Pensamiento Constitucional, Pontificia Universidad Católica del Perú, 335 (2008).

10 Lo que calificará la existencia de un derecho social como derecho no es simplemente la conducta cumplida por el Estado, sino la existencia de algún poder jurídico de actuar del titular del derecho en el caso de incumplimiento de la obligación debida. Considerar a un derecho económico, social o cultural como derecho es posible únicamente si -al menos en alguna medidael titular/acreedor está en condiciones de producir mediante una demanda o queja, el dictado de una sentencia que imponga el cumplimiento de la obligación que constituye el objeto de su derecho 25. VÍCTOR ABRAMOVICH \& CHRISTIAN COURTIS, Hacia la exigibilidad de los derechos económicos, sociales y culturales. Estándares internacionales y criterios de aplicación ante los tribunales locales (1997). Disponible en: http://miguelcarbonell.com/artman/uploads/1/Exigibilidad_de_los_DESC_-_ Abramovich.pdf

11 FRANCISCO ZÚÑIGA, Derechos económicos, sociales y culturales: apuntes acerca de la naturaleza y justiciabilidad de los derechos fundamentales, Revista Pensamiento Constitucional, Pontificia Universidad Católica del Perú, 335 (2008).

12 IVETTE GÓMEZ BUSTOS, La acción colectiva del agua en Colombia y el referendo como acercamiento de democracia directa, Revista Análisis Político, 80 (2014). 
13 Hasta 2016 la Corte Constitucional ha proferido 32 sentencias sobre el derecho al mínimo vital del agua potable. ÁLVARO OSORIO SIERRA, ANTONIO EMBID IRUJO \& JORGE IVÁN HURTADO MORA, Régimen de los usos del agua. Análisis desde la perspectiva jurídica, 87-88 (Universidad de Zaragoza y Universidad Externado de Colombia, Bogotá, 2016).

14 ALBERTO CARDONA LÓPEZ, Consideraciones sobre el sector de agua potable y saneamiento básico en Colombia, (2010).Disponible en: https://colaboracion.dnp.gov.co/CDT/Estudios\%20Econmicos/Consideraciones\%20sobre \%20el\%20Sector\%20de\%20agua\%20potable\%20y\%20saneamiento\%20b\%C3\%A1sico.pdf

\section{JORGE GONZÁLEZ GONZÁLEZ, El acceso al agua potable como derecho humano. Su dimensión internacional, Club} Universitario, 27-133 (2014).

16 En este caso, en relación a la desigualdad en el reparto de los recursos hídricos "algunas áreas geográficas padecen una falta crónica de agua, otras disponen de los recursos necesarios, e incluso existen las que sufren una súper abundancia de agua continua o estacionaria". HABIB AYEB, Agua y poder. Geopolitica de los recursos hidráulicos en Oriente Próximo, Bella Terra 13 (2001).

17 El consumo promedio de agua por habitante no es igual en todos los países del mundo. Por ejemplo "en Estados Unidos el consumo es de seiscientos litros per cápita, mientras que en África, los habitantes sobreviven con menos de 20 litros de agua al día". JORGE GONZÁLEZ GONZÁLEZ, El acceso al agua potable como derecho humano. Su dimensión internacional, Club Universitario, 27-133 (2014).

18 En la Sentencia T-546 de 2009 de la Corte Constitucional de Colombia, se puede observar la regulación que la Corte le da al mínimo vital de agua potable y el respaldo del derecho humano al agua potable a partir de instrumentos internacionales en virtud del bloque de constitucionalidad. Al respecto indica:"A juicio de la Sala, no en todo caso de incumplimiento es válido suspender los servicios públicos domiciliarios, en el sentido de cortar totalmente el suministro de los mismos. Si el incumplimiento es involuntario u obedece a una fuerza insuperable; si, además, el domicilio a que se destinan está habitado por personas que merecen una especial protección constitucional; si el servicio es de aquellos indispensables para garantizar otros derechos fundamentales como la vida, la igualdad, la dignidad o la salud; y si, por último, se dan las condiciones establecidas en la ley para la suspensión, lo que debe suspenderse es la forma de prestar el servicio público. Es decir, debe cambiar la forma en que se suministra el servicio y ofrecerle al destinatario final unas cantidades mínimas básicas e indispensables, en este caso, de agua potable.Con todo, esas cantidades mínimas deben ser fijadas por la Empresa de Servicios Públicos, en consideración a la cantidad de personas que habiten en el domicilio y con sujeción a criterios aceptables desde el punto de vista de su capacidad para garantizar los derechos a la vida, la salud y la dignidad de los niños que habiten en ella. Solo para efectos de ilustrar cómo pueden ser adoptadas esas medidas mínimas de agua potable, conviene señalar lo manifestado en el Informe del Alto Comisionado de las Naciones Unidas para los Derechos Humanos sobre el alcance y el contenido de las obligaciones pertinentes en materia de derechos humanos relacionadas con el acceso equitativo al agua potable y el saneamiento que imponen los instrumentos internacionales de derechos humanos. De acuerdo con este Informe, "si bien incumbe a cada país determinar el volumen mínimo razonable de agua necesaria para satisfacer los usos personales y domésticos, las cifras suministradas en las publicaciones de la Organización Mundial de la Salud (OMS) pueden servir de orientación útil. Por consiguiente, se necesitan entre 50 y 100 litros de agua por persona por día para asegurar la satisfacción de todas las necesidades de salud (31). El umbral de 25 litros por persona por día representa el nivel mínimo para mantener la vida, pero esta cantidad plantea problemas de salud, ya que es insuficiente para atender las necesidades de higiene básica y consumo (32). En los casos de emergencia, tales como desastres naturales, conflictos o situaciones después de los conflictos, el Manual del Proyecto Esfera sugiere un abastecimiento básico de 7,5 a 15 litros mínimos por persona y por día, ya que puede no haber suficiente agua disponible para atender a todos los usos personales y domésticos (33). Estas diversas cantidades son indicativas, ya que pueden cambiar con arreglo a un contexto en particular y pueden diferir en el caso de algunos grupos, debido a su estado de salud, trabajo, condiciones climáticas, exigencias culturales u otros factores".

19 DIANA MARGARITA MIGUEZ CARAMÉS, Gestión integrada de recursos hidricos en Uruguay en el contexto internacional, Revista del Laboratorio Tecnológico del Uruguay, 72 (2015).

20 Se entiende por estrés hídrico "el desequilibrio entre el consumo y el agua disponible". MANUEL GUERRERO E ISAAC SHIFTER, La huella del agua 63 (Fondo de Cultura Económica, México, 2012).

21 JORGE GONZÁLEZ GONZÁLEZ, El acceso al agua potable como derecho humano. Su dimensión internacional, Club Universitario, 133 (2014)

22 RED-DESC. Sección 1: La historia del PF-PIDESC, 4 (2010). Disponible en: https://www.escr-net.org/es/recursos/secci on-1-historia-del-pf-pidesc

23 "La Sra. Catarina de Albuquerque es la primera Relatora Especial de las Naciones Unidas sobre el derecho al agua potable y al saneamiento (anteriormente experta independiente). Fue nombrada por el Consejo de Derechos Humanos en septiembre de 2008 
e inició el ejercicio de su mandato el 1 de noviembre de ese mismo año. De 2004 a 2008 presidió las negociaciones del Protocolo Facultativo del Pacto Internacional de derechos económicos, sociales y culturales, que la Asamblea General de las Naciones Unidas aprobó por consenso el 10 de diciembre de 2008. La Sra. de Albuquerque es profesora invitada en las facultades de Derecho de las Universidades de Coimbra y Braga en Portugal y se desempeña como Asesora Jurídica Superior de la Oficina de Documentación y Derecho Comparado, una institución independiente bajo la dirección de la Fiscalía General”. UN, Sra. Catarina de Albuquerque, Relatora Especial sobre el derecho humano al agua potable y al saneamiento. Disponible en: http://www.ohchr.org/SP/Issues/W aterAndSanitation/SRWater/Pages/CatarinaDeAlbuquerque.aspx (n. d.).

24 Resolución de la Asamblea General de 10 de diciembre de 2008, A/RES/63/117.

25 LAURA SALAMERO TEIXIDÓ, La protección de los derechos sociales en el ámbito de las Naciones Unidas, 55 (CivitasThomson Reuters, Pamplona, España, 2012).

26 El hecho de que el Pacto Internacional de Derechos Económicos, Sociales y Culturales solo previera un sistema de informes, con respecto al sistema de quejas interestatales y comunicaciones individuales previstas en el Pacto Internacional de Derechos Civiles y Políticos, supuso una honda fractura en el principio de indivisibilidad e interdependencia de las dos categorías de derechos según se proclamaba en la Declaración Universal de Derechos Humanos. Esta ruptura pretende ser finalmente enmendada mediante la adopción el 10 de diciembre de 2008 por la Asamblea General de Naciones Unidas del Protocolo Facultativo del PIDESC. La aprobación de este Protocolo Facultativo representa un hito en la trayectoria de la protección de los derechos económicos, sociales y culturales en el ámbito internacional, pues supone un avance formal para su verdadera equiparación con los derechos civiles y políticos, al reconocerles un grado parejo de justiciabilidad. LAURA SALAMERO TEIXIDÓ, La protección de los derechos sociales en el ámbito de las Naciones Unidas. Aranzadi, 55 (Civitas-Thomson Reuters, Pamplona, España, 2012).

27 Naciones Unidas, Derechos Humanos, Oficia del Alto Comisionado para los Derechos Humanos, Oficina Regional para América del Sur, Protocolo Facultativo del Pacto Internacional de Derechos Económicos, Sociales y Culturales. Disponible en: http:/ /derechoshumanos.pe/wp-content/woo_uploads/congreso/Folleto_Informativo_Protocolo_Facultativo_PIDESC.pdf

28 Es decir no para consumos suntuarios o que superen el mínimo vital.

29 ELISA PÉREZ DE LOS COBOS, La defensa del Derecho al Agua como derecho Colectivo desde su perspectiva Ambiental, Revista Catalana de Derecho Ambiental, VI, 2, 11 (2015).

30 HUGO ALFONSO HERNÁNDEZ \& JHON MENDEZ SAYAGO, Viabilidad económica del minimo vital de agua potable en la ciudad de Bogotá D. C. Revista Producción + Limpia, 8, 1, 104 (Enero-Junio 2013). .

31 Los estratos socioeconómicos son una forma de clasificar las viviendas con el único fin de focalizar subsidios para los servicios públicos domiciliarios. Los inmuebles residenciales que están ubicados en las categorías 1, 2 y 3 gozan de subsidios o descuentos en sus facturas por el consumo en servicios públicos domiciliarios, que para el caso que nos ocupa es el servicio de acueducto. Por otra parte, los inmuebles clasificados en estrato 5 y 6 tendrán un sobreprecio en su factura para ayudar en el pago de los servicios públicos domiciliarios a los usuarios ubicados en los inmuebles de categorías 1, 2 y 3. Vale resaltar que el Estado también entra a subsidiar a los usuarios de estas últimas categorías mencionadas.

32 HUGO ALFONSO HERNÁNDEZ \& JHON MÉNDEZ SAYAGO, Viabilidad económica del mínimo vital de agua potable en la ciudad de Bogotá D. C., Revista Producción + Limpia. 8, 1, 104 (Enero-Junio 2013).

33 LUIS BOHÓRQUEZ CALDERA, Bioética del derecho al agua potable, AGO.USB, 325-585 (2015).

34 El artículo 99.5 de la Ley 142 de 1994 establece que "los subsidios no excederán, en ningún caso, del valor de los consumos básicos o de subsistencia. Los alcaldes y los concejales tomarán las medidas que a cada uno corresponda para crear en el presupuesto municipal y ejecutar apropiaciones para subsidiar los consumos básicos de acueducto [y saneamiento básico] de los usuarios de menores recursos y extender la cobertura y mejorar la calidad de los servicios de agua potable y saneamiento básico, dando prioridad a esas apropiaciones, dentro de las posibilidades del municipio, sobre otros gastos que no sean indispensables para el funcionamiento de este. La infracción de este deber dará lugar a sanción disciplinaria”.

35 Según la Comisión de Regulación de Agua Potable y Saneamiento básico de Colombia (CRA), estos son los siguientes niveles de consumo básico para el país: $15 \mathrm{~m}^{3} /$ suscriptor/mes para municipios ubicados en clima cálido, $13 \mathrm{~m}^{3} /$ suscriptor/mes para municipios ubicados en clima templado y $11 \mathrm{~m}^{3} /$ suscriptor/mes para municipios ubicados en clima frío. CRA, Clasificación temática. Consumo básico de agua. Disponible en: http://www.cra.gov.co/es/novedades/noticias/23739-conoce-el-nuevo-consu mo-b 
36 Corte Constitucional de Colombia, Sala Plena, Sentencia T-055 de 2011, Expediente T-2.804.492, magistrado ponente: Jorge Iván Palacio Palacio.

37 Corte Constitucional de Colombia, Sala Plena, Sentencia T-055 de 2011, Expediente T-2.804.492, magistrado ponente: Jorge Iván Palacio Palacio.

38 LUIS AMADOR CABRA, Los servicios públicos frente a las reformas económicas en Colombia, 410 (Universidad Externado de Colombia, Bogotá, 2011).

39 MAUDE BARLOW, El derecho al agua. Agua, un derecho y no una mercancía. Propuestas de la sociedad civil para el modelo público de agua, 109 (Icaria, Barcelona, España, 2009).

40 MILTON CÉSAR JIMÉNEZ RAMÍREZ, El minimo vital de acceso al agua potable frente a las empresas de servicios públicos en Colombia: personas en situación de debilidad manifiesta vs mercado de servicios públicos, Revista Pensamiento Jurídico, 38, 117 (2013).

41 MILTON CÉSAR JIMÉNEZ RAMÍREZ, El minimo vital de acceso al agua potable frente a las empresas de servicios públicos en Colombia: personas en situación de debilidad manifiesta vs mercado de servicios públicos, Revista Pensamiento Jurídico, 38, 119 (2013).

42 SAMUEL RODRÍGUEZ FERNÁNDEZ, La tutela penal del agua. El bien jurídico protegido, 88 (Dykinson, Madrid, España, 2009).

43 Las inversiones en infraestructura para la provisión del servicio de acueducto y alcantarillado son financiadas por el Estado.

44 La demanda también cuenta con subsidios, que es el descuento en la tarifa para que los usuarios de menores ingresos puedan pagar los servicios de agua, alcantarillado y aseo.

\section{Licencia Creative Commons CC BY 4.0}

Para citar este articulo/To cite this article: Echeverría-Molina, Judith \& Anaya-Morales, Shiley, El derecho humano al agua potable en Colombia: decisiones del Estado y de los particulares, 136 Vniversitas, 1-14 (2018). https://doi.org/10.11144/Javeriana.vj136.dhap 